N $\quad 80 \quad 2738$ 3 X-643-69-402

NAs $\frac{10-\operatorname{Tas} \frac{2}{2}}{2} 63993$

\title{
THERMAL AND TIDAL EFFECT ON THE ROTATION OF MERCURY
}

HAN-SHOU LIU

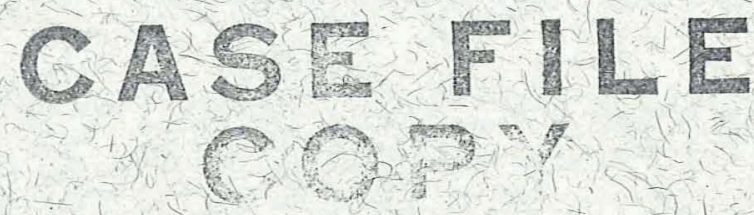

AUGUST 1969

GSFC GODDARD SPACE FLIGHT CENTER GREENBELT, MARYLAND 
THERMAL AND TIDAL EFFECT ON

THE ROTATION OF MERCURY

Han-Shou Liu

August 1969

GODDARD SPACE FLIGHT CENTER

Greenbelt, Maryland 


\title{
THERMAL AND TIDAL EFFECT ON
}

THE ROTATION OF MERCURY

\author{
Han-Shou Liu \\ Goddard Space Flight Center \\ Greenbelt, Maryland
}

\begin{abstract}
It is shown that the influences of the thermal and tidal effects on Mercury's libration are in equilibrium with the periods of rotation and revolution of Mercury locked in the 3:2 resonant state. The suggestion by Liu that the solar gravitational couple on the thermal bulges accelerates Mercury's rotation is investigated and the production of mechanical energy to balance the dissipation of the bodily tides is discussed. It is possible for Mercury to rotate with two bulges as a solar thermal engine; the tidal effect causes this engine to function and its maximum power is close to $10^{16}$ ergs per second.
\end{abstract}




\section{CONTENTS}

\section{$\underline{\text { Page }}$}

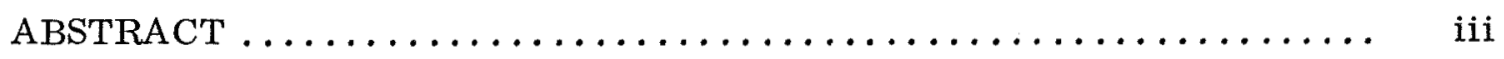

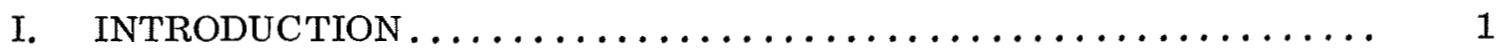

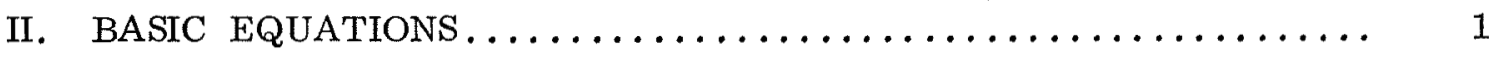

III. ANALYSIS $\ldots \ldots \ldots \ldots \ldots \ldots \ldots \ldots \ldots \ldots \ldots \ldots \ldots \ldots \ldots \ldots \ldots \ldots \ldots \ldots, 2$

IV. CONCLUDING REMARKS $\ldots \ldots \ldots \ldots \ldots \ldots \ldots \ldots \ldots \ldots \ldots \ldots \ldots \ldots \ldots, 6$

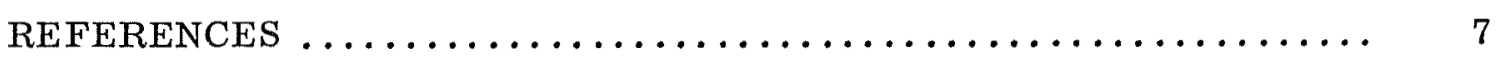




\title{
THERMAL AND TIDAL EFFECT ON
}

\author{
THE ROTATION OF MERCURY
}

\section{INTRODUCTION}

Liu (Liu, 1969; Liu, 1968) has suggested that the trapping of Mercury's rotational period into a $3: 2$ resonance lock with its orbital period was originally affected by the thermal contraction of the figure of Mercury during solidification. In the present paper the analysis of the contribution of the two thermal bulges to the dynamic stabilization of the planet's rotation is given. Attention is focused on the balance of the influence of the tidal and thermal effect on the libration of Mercury after solidification.

\section{BASIC EQUATIONS}

After solidification, the thermal contraction of the figure of Mercury from loss of heat must be exceedingly small and the thermal bulges considered by Liu (Liu, 1969; Liu, 1968) can grow because the apparent circulational motion at successive perihelia has been converted to a librational motion. The thermoelastic variation with time of the fractional difference in Mercury's equatorial moments of inertia about the state of hydrostatic equilibrium is

$$
\frac{B(t)-A(t)}{C(t)}=\frac{B(h)-A(h)}{C(h)}+\frac{3\left(2-5 \beta^{3}+3 \beta^{5}\right)}{100\left(1+\beta+\beta^{2}\right)} \cdot a \cdot \Delta T
$$

in which $\alpha$ is the coefficient of linear thermal expansion, $\Delta \mathrm{T}$ the difference in surface temperature between the regions around the perihelion and the aphelion axes of Mercury, and $\beta=(\Re \mathrm{m}-\mathrm{y}) / \Omega_{\mathrm{m}}$ where $R \mathrm{~m}$ is Mercury's radius and $\mathrm{y}$ the depth of solar heating. The value of $[B(h)-A(h)] / C(h)$ has been estimated as $3 \times 10^{-6}$ and the rate of increasing of $[B(t)-A(t)] / C(t)$ is about $10^{-21}$ Sec. ${ }^{-1}$ (Liu and $\mathrm{O}^{\prime}$ Keefe, 1965; Liu, 1968; Liu, 1969). Since the local temperature regimes depend strongly on the orientation and the orbital position of Mercury relative to the Sun, a temperature phase effect is apparent with a phase lead of $35.5^{\circ}$ ( $\left.\mathrm{Liu}, 1966 \mathrm{~b}\right)$. Consequently, the angle between the axes of $\mathrm{A}(\mathrm{h})$ and the two thermal bulges is about $0.6^{\circ}$.

In considering the bodily tidal torque we have recourse to the estimation by Jeffreys (Jeffreys, 1959). 


$$
\mathrm{N}=\frac{18}{5} \cdot \mathrm{k} \pi \mathrm{G} \rho \frac{\mathrm{M}_{\mathrm{s}}^{2}}{\mathrm{M}_{\mathrm{m}}^{2}} \cdot \frac{\mathrm{Rm}^{6}(1+\mathrm{e} \cos \mathrm{f})^{6}}{\mathrm{a}^{6}(1-\mathrm{e})} \cdot \mathrm{C}_{(\mathrm{h})} \cdot \operatorname{Sin} 2 \epsilon
$$

where $\mathrm{G}$ is the gravitational constant, $\rho$ density, Ms mass of the Sun, Mm mass of Mercury, $f$ the true anomaly, a the semimajor axis, e the orbital eccentricity, $\epsilon$ the phase lag of the conventional equilibrium tides and $\mathrm{k}$ the Love number.

The orientation of Mercury relative to the Sun, $\varphi$, is then governed by (Liu and $O^{\prime}$ Keefe, 1965)

$\frac{d^{2} \varphi}{d f^{2}}-\frac{2 e \sin f}{1+e \cos f}\left(\frac{d \varphi}{d f}+1\right)+\frac{3\left(\lambda_{(h)}+\Delta \lambda\right)}{2(1+e \cos f)} \sin 2 \varphi=-\frac{N}{n^{2} C_{(h)}} \cdot \frac{\left(1-e^{2}\right)^{3}}{(1+e \cos f)^{4}}$

where

$$
\begin{gathered}
\lambda_{(h)}=\frac{B(h)-A(h)}{C(h)} \\
\Delta \lambda=\frac{3}{20}\left(\frac{y}{R_{m}}\right)^{2} \cdot \alpha \cdot \Delta T
\end{gathered}
$$

and $\mathrm{n}$ is the mean orbital angular velocity.

\section{ANALYSIS}

Let us choose the time $\tau$ reckoned from perihelion and related to the orbital period divided by $2 \pi$

$$
\tau_{(f)}=2 \tan ^{-1}\left(\frac{1-e}{1+e}\right)^{\frac{1}{2}} \tan \frac{f}{2}-\frac{e\left(1-e^{2}\right)^{\frac{1}{2}} \sin f}{1+e \cos f}
$$

As a new unknown function, we take the angle of rotation $\psi$ between the axis of $A(h)$ and the radius vector of the perihelion 


$$
\psi=f+\varphi
$$

Equation (3) takes the form

$$
\frac{d^{2} \psi}{d \tau^{2}}+\frac{3\left(\lambda_{(h)}+\Delta \lambda\right)}{2} \cdot \frac{(1+e \cos f)^{3}}{\left(1-e^{2}\right)^{3}} \sin 2(\psi-f)=-\frac{N}{n^{2} C_{(h)}}
$$

The solution of Equation (6) may be sought in the form

$$
\psi=\Omega \tau+\delta
$$

where $\Omega$ is a constant and $\delta$ is an unknown function. The resonances occur at $2 \Omega=\mathrm{m}$ if $\mathrm{m}$ is an integer and $\delta \mathrm{is}$, then, the angle of libration. To obtain an approximate expression for the libration of Mercury we may average it over a period of $2 \pi$ for $m=3$. If the imperfection of elasticity adopted for the Earth (Jeffreys, 1959) is supposed to hold right up to the surface of Mercury, Equation (6) becomes

$$
\frac{\mathrm{d}^{2} \delta}{\mathrm{d} \tau^{2}}+\frac{2 \cdot 1}{2}\left(\lambda_{(\mathrm{h})}+\Delta \lambda\right) \operatorname{Sin} 2 \delta=-10^{-11}
$$

In the derivation of Equation (7), the following values were adopted:

$$
\begin{aligned}
\mathrm{e} & =0.2 \\
\mathrm{k} & =0.02 \\
2 \epsilon & =0.005 \\
\mathrm{G} & =6.7 \times 10^{-8} \mathrm{dyn} \cdot \mathrm{Cm}^{2} \cdot \mathrm{g}^{-2} \\
\rho & =5.0 \mathrm{~g} \cdot \mathrm{Cm}^{-3} \\
\mathrm{n} & =1.2 \times 10^{-6} \mathrm{rad} \cdot \cdot \mathrm{Sec}^{-1} \\
\mathrm{Ms} / \mathrm{Mm} & =6.0 \times 10^{6} \\
\Re \mathrm{m} / \mathrm{a} & =4.1 \times 10^{-5}
\end{aligned}
$$


The average process also included the reversal of the tidal torque in direction during perihelion passage (Peale and Gold, 1965; Liu, 1966b).

The first integral of Equation (7) is

$$
\frac{1}{2}\left(\frac{\mathrm{d} \delta}{\mathrm{d} \tau}\right)^{2}-\frac{2 \cdot 1}{4}\left(\lambda_{(\mathrm{h})}+\Delta \lambda\right) \cos 2 \delta=-10^{-11} \cdot \delta+\mathrm{E}_{0}
$$

where $\mathrm{E}_{0}$ is a constant.

Differentiating Equation (8) with respect to $t$, the result is

$$
\begin{aligned}
\frac{\mathrm{d}}{\mathrm{dt}}\left[\frac{1}{2}\left(\frac{\mathrm{d} \delta}{\mathrm{dt}}\right)^{2}-\frac{2 \cdot 1}{4} \mathrm{n}^{2} \cdot \lambda_{(\mathrm{h})} \cdot \cos 2 \delta\right] \\
\quad=\frac{2 \cdot 1}{4} \mathrm{n}^{2} \cdot \frac{\mathrm{d}(\Delta \lambda)}{\mathrm{dt}} \cdot \operatorname{Cos} 2 \delta-10^{-11} \cdot \mathrm{n}^{2} \cdot \frac{\mathrm{d} \delta}{\mathrm{dt}}-\frac{2 \cdot 1}{2} \mathrm{n}^{2} \cdot \Delta \lambda \cdot \operatorname{Sin} 2 \delta \cdot \frac{\mathrm{d} \delta}{\mathrm{dt}}
\end{aligned}
$$

The first and second term in the left side of Equation (9) correspond to kinetic and potential energies, respectively, of the system. In order to maintain the prescribed librational motion, energy must be added to the system because of tidal dissipation. Earlier investigations (Goldreich and Peale, 1968; Goldreich and Peale, 1966a; Goldreich and Peale, 1966b; Colombo, 1965; Colombo and Shapiro, 1966; Jefferys, 1966; Laslett and Sessler, 1966; Liu, 1966a; Liu and O'Keefe, 1965; Lutze and Abbitt, 1969) have shown that a sufficient deviation from axial symmetry would stabilize the libration of Mercury at this resonance state. Therefore the instantaneous deformation of the figure of Mercury into a hydrostatic equilibrium shape with $\lambda(\mathrm{h})=[\mathrm{B}(\mathrm{h})-\mathrm{A}(\mathrm{h})] / \mathrm{C}(\mathrm{h})=3 \times 10^{-6}$ (Liu, $1969)$ is sufficient to stabilize this resonance against the disruptive influence of the tidal or any other effects. However, the dynamic behavior and the evolution of the tidal or thermal process near this resonance condition are still unknown. To explain the physical feature of the locked-in motion, Liu (Liu, 1969; Liu, 1968) has proposed that two bulges which arise on Mercury's surface from solar heating after the rotation becomes commensurable would increase the strength of the resonance and tend to prevent drifting out. This fact can be seen by computing the rate of working of the thermal and tidal effects. Equation (9) can be written in the form, 


$$
\frac{d E}{d t}=\left(\frac{d E}{d t}\right)_{\text {thermal expansion }}+\left(\frac{d E}{d t}\right)_{t i d a l \text { effect }}+\left(\frac{d E}{d t}\right)_{\text {thermal bulges }}
$$

where

$$
\begin{aligned}
& \left(\frac{d E}{d t}\right)_{\text {thermal expansion }}=\frac{2 \cdot 1}{4} n^{2} \cdot \operatorname{Cos} 2 \delta \cdot \frac{d(\Delta \lambda)}{d t} \cdot C_{(t)} \\
& \left(\frac{d E}{d t}\right)_{t i d a 1 \text { effect }}=-10^{-11} \cdot n^{2} \cdot \frac{d \delta}{d t} \cdot C_{(t)} \\
& \left(\frac{d E}{d t}\right)_{\text {thermal bulges }}=-\frac{2 \cdot 1}{2} \cdot n^{2} \cdot \Delta \lambda \cdot \operatorname{Sin} 2 \delta \cdot \frac{d \delta}{d t} \cdot C_{(t)}
\end{aligned}
$$

For small angle of libration $\delta, d(\Delta \lambda) / d t=10^{-21} \mathrm{Sec}^{-1}$, and $\mathrm{d} \delta / \mathrm{dt} \leq 10^{-3} \mathrm{n}$, we obtain

$$
\begin{aligned}
& \left(\frac{d E}{d t}\right)_{\text {thermal expansion }}=0\left(10^{10} \text { ergs. Sec. }{ }^{-1}\right) \\
& \left(\frac{d E}{d t}\right)_{\text {tidal effect }}=-0\left(10^{10} \text { ergs. Sec. }{ }^{-1}\right)
\end{aligned}
$$

The thermal expansion effect works against the tidal effect on Mercury's libration at a rate of $10^{10} \mathrm{ergs} \mathrm{Sec}^{-1}$. It is of the same order of magnitude as the rate of the librational dissipation for the bodily tidal friction of Mercury. This result does not exclude entirely the possibility of equilibrium between the thermal expansion and the tidal effects but it must be admitted that the impression remains that the tidal effect is the larger and that the rotation of Mercury is being retarded. For this reason, one is still inclined to be dissatisfied with the fact that the Liu-O'Keefe theory depends on a chance. This misunderstanding, however, can be easily cleared up. The most important feature of the rotational lock for the present case is that the main component is the one corresponding to the mechanical work done by the couple of thermal bulges. The accelerating 
couple exerted by solar attraction of Mercury's thermal bulges has been mentioned. As Mercury seems to be the same age as the solar system, about 4.5 billion years old, these bulges may well have existed for a large fraction of this time. Thus the value of $\Delta \lambda$ at present time is about $10^{-5}$. If the deviation of the axis of the two thermal bulges from line of centers at positions $f=0^{\circ}$ and $\pm 45^{\circ}$ increased $0.6^{\circ}$ in the clockwise direction as seen from the north, the rate of working by the thermal bulges is

$$
\left(\frac{\mathrm{dE}}{\mathrm{dt}}\right)_{\text {thermal bulges }}=O\left(10^{14} \text { ergs. Sec. }{ }^{-1}\right)
$$

which is about four orders of magnitude larger than that of the bodily tidal effect. As the amplitude of this deviation increases due to tidal effect, it will exert a couple which becomes progressively more effective in reducing the retardation until the thermal and tidal effects are in equilibrium with the orbital and rotational periods locked at the 3:2 resonant state. For an offset of the thermal bulges of $\pi / 4$ from the radius vector at perihelion, this rate of working should multiply a factor of 100. Therefore Mercury may rotate as a celestial thermal engine; the tidal effect causes the engine to function and its maximum power is close to $10^{16}$ ergs. $\mathrm{Sec}^{-1}$. It should be noted that the efficiency required of the planet Mercury as a thermal engine is very small. The maximum rate of working by the thermal bulges is only the small fraction $10^{-7}$ of the total rate at which effective heat energy is received from the Sun. The efficiency of an ideal thermal engine working on a temperature drop of $1 \mathrm{deg}$. $\mathrm{C}$ at $300 \mathrm{deg} . \mathrm{K}$ is about $10^{-3}$ so there is a considerable margin to allow for irreversible processes or heat loss.

\section{CONCLUDING REMARKS}

We have shown that the influences of the thermal and tidal effect on Mercury's libration are in equilibrium with the periods of rotation and revolution of Mercury locked in the 3:2 state. With regard to the interaction of the thermal effect, tidal friction and gravitation, it is concluded that the two thermal bulges on Mercury's surface contribute dynamically to the stabilization of the planet's rotation. 


\section{REFERENCES}

Colombo, G., 1965, The Rotation of the Planet Mercury, Nature 208, 575.

Colombo, G. and Shapiro, I. I., 1966, The Rotation of the Planet Mercury, Ap. J. $145,296$.

Goldreich, P. and Peale, S. J., 1968, The Dynamics of Planetary Rotations, Annual Review of Astronomy and Astrophysics, 6, 287.

Goldreich, P. and Peale, S. J., 1966a, Resonant Spin States in the Solar System, Nature 209, 1078.

Goldreich, P. and Peale, S. J., 1966b, Spin-Orbit Coupling in the Solar System, The Astronomical J. 1341, 425.

Jefferys, W. H., 1966, On the Rotation of the Planet Mercury, Science 152, 201.

Jeffreys, H., 1959, The Earth, Cambridge University Press.

Laslett, L. J. and Sessler, A. M., 1966, Rotation of Mercury: Theoretical Analysis of the Dynamics of a Rigid Ellipsoidal Planet, Science 151, 1384.

Liu, H. S., 1969, On the Figure of Mercury, Celestial Mechanics, 2, 31.

Liu, H. S., 1968, Mercury Has Two Thermal Bulges, Science 159, 306.

Liu, H. S., 1966a, The Libration of Mercury, J. Geophys. Res. 71, 3099.

Liu, H. S., 1966b, Unpublished Results.

Liu, H. S. and O'Keefe, J. A., 1965, Theory of Rotation for the Planet Mercury, Science 150, 1717 .

Lutze, F. H. and Abbitt, M. W., 1969, Rotational Locks for Near-Symmetric Satellites, Celestial Mechanics 1, 31.

Peale, S. J. and Gold, T., 1965, Rotation of the Planet Mercury, Nature 206, 1240. 